\begin{tabular}{|c|c|}
\hline Citation/Reference & $\begin{array}{l}\text { Devy Widjaja, Steven Vandeput, Sabine Van Huffel, André E Aubert, } \\
\text { (2015), } \\
\text { Cardiovascular Autonomic Adaptation in Lunar and Martian Gravity } \\
\text { during Parabolic Flight } \\
\text { European Journal of Applied Physiology, } 115 \text { (6), 1205-1218. }\end{array}$ \\
\hline Archived version & $\begin{array}{l}\text { Author manuscript: the content is identical to the content of the published } \\
\text { paper, but without the final typesetting by the publisher }\end{array}$ \\
\hline Published version & $\begin{array}{l}\text { insert link to the published version of your paper } \\
\text { http://dx.doi.org/10.1007/s00421-015-3118-8 }\end{array}$ \\
\hline Journal homepage & http://www.springer.com/biomed/human+physiology/journal/421 \\
\hline Author contact & Devy.widjaja@esat.kuleuven.be \\
\hline IR & https://lirias.kuleuven.be/handle/123456789/488730 \\
\hline
\end{tabular}




\title{
Cardiovascular Autonomic Adaptation in Lunar and Martian Gravity during Parabolic Flight
}

\author{
Devy Widjaja · Steven Vandeput - Sabine Van Huffel • André E. Aubert
}

Received: date / Accepted: date

\begin{abstract}
Purpose: Weightlessness has a well-known effect on the autonomic control of the cardiovascular system. With future missions to Mars in mind, it is important to know what the effect of partial gravity is on the human body. We aim to study the autonomic response of the cardiovascular system to partial gravity levels, as present on the Moon and on Mars, during parabolic flight.

Methods: ECG and blood pressure were continuously recorded during parabolic flight. A temporal analysis of blood pressure and heart rate to changing gravity was conducted to study the dynamic response. Additionally, cardiovascular autonomic control was quantified by means of heart rate (HR) and blood pressure (BP) variability measures.

Results: Zero and lunar gravity presented a biphasic cardiovascular response, while a triphasic response was noted during martian gravity. Heart rate and blood pressure are positively correlated with gravity, while the general variability of $\mathrm{HR}$ and $\mathrm{BP}$, as well as vagal indices showed negative correlations with increasing gravity. However, the increase in vagal modulation during weightlessness is not in proportion when compared to the increase during partial gravity.
\end{abstract}

D. Widjaja, S. Vandeput, S. Van Huffel

KU Leuven, Department of Electrical Engineering (ESAT) - STADIUS iMinds Medical Information Technologies

Kasteelpark Arenberg 10, box 2446

3001 Leuven, Belgium

E-mail: devy.widjaja@esat.kuleuven.be

A.E. Aubert

KU Leuven, Laboratory of Experimental Cardiology, University Hospital Gasthuisberg

Herestraat 49

3000 Leuven, Belgium
Conclusions: Correlations were found between the gravity level and modulations in the autonomic nervous system during parabolic flight. Nevertheless, with future Mars missions in mind, more studies are needed to use these findings to develop appropriate countermeasures.

Keywords parabolic flight · lunar gravity · microgravity · martian gravity $\cdot$ heart rate variability $\cdot$ blood pressure variability

\section{Introduction}

It is well-known that long-term space flight poses several risks for the astronauts due to changes in radiation, the exposure to microgravity, and the prolonged confinement (Aubert et al. 2005). Additionally, when they return to earth, they may suffer from postflight orthostatic intolerance (Sides et al, 2005). Although the underlying mechanisms are not fully understood (Buckey Jr et al 1996), it has been identified that the autonomic control of the cardiovascular system plays an important role in the development of orthostatic intolerance (Aubert et al, 2005 Baevsky et al, 2007; Cooke et al, 2000).

In the last decades, there has been an increasing interest to travel to Mars. In order to prepare for a manned space flight to Mars, a 105-day pilot study and a 520-day study has been carried out to simulate a mission to Mars, where a small crew was confined for 520 days, hereby mimicking the time necessary to travel back and forth to Mars and the tasks they would have to perform. During these preparation studies, the cardiovascular response to the prolonged confinement was evaluated (Vigo et al 2012, 2013, Wan et al, 2011). Only the reduction in gravity 
was not simulated. Although many studies have already investigated how the human body behaves during microgravity (Aubert et al, 2005), its response to reduced gravity levels, as on Mars $(0.38 \mathrm{~g})$ and the Moon $(0.16 g)$, is not yet clear. In recent studies, Moon and Mars gravity fields were simulated using lower body positive pressure (Evans et al, 2013; Kostas et al, 2014). While standing, fluid shifts from the chest were shown at different body weights, eliciting changes in regulatory responses. In this study, parabolic flights are used to assess cardiovascular autonomic functioning during changing gravity conditions, where periods of reduced gravity are preceded and followed by hypergravity phases (1.6 - $1.8 \mathrm{~g})$. Previous studies already showed that during hypergravity, blood is pulled towards the lower extremities, leading to a reduction in venous return and stroke volume (Le Rolle et al, 2008), while the opposite occurs during microgravity (Beckers et al, 2003; Mukai et al, 1991). These hemodynamic changes lead to vagal and sympathetic modulations via the baroreflex and cardiopulmonary reflex (Beckers et al, 2003: Linnarsson et al, 1996: Liu et al, 2011: Pump et al, 1999: Verheyden et al, 2005).

The purpose of this study is to assess the autonomic response of the cardiovascular system to lunar, martian and zero gravity conditions obtained during parabolic flights. The functioning of the autonomic nervous system is quantified by means of heart rate (HRV) and blood pressure variability (BPV). Analyses of HRV and BPV provide, in an easy and non-invasive way, information on autonomic control of the cardiovascular system, and has been used to study the autonomic response to weightlessness induced by parabolic flights (Beckers et al, 2003: Liu et al, 2011; Mukai et al, 1991; Seps et al, 2002, Verheyden et al, 2005). We, now, aim to investigate whether a (linear) relation exists between measures of HRV and BPV on the one hand, and the gravity level on the other hand. Now, the latter not only comprises hypergravity, normogravity and microgravity conditions; with the Joint European Partial-g parabolic flight campaigns, cardiac and blood pressure recordings could be collected during martian and lunar gravity. Preliminary results of these analyses were presented at the Joint Life Science Meeting 'Life in Space for Life on Earth' in Abderdeen, UK in June 2012 (Aerts et al, 2012) and the 19th IAA Humans in Space Symposium in Cologne, Germany in July 2013 (Widjaja et al, 2013).

\section{Data}

\subsection{Participants}

Fourteen healthy, non-smoking, male subjects (mean \pm SD; age: $28.4 \pm 3.7 \mathrm{yr}$; height: $178.9 \pm 5.5 \mathrm{~cm}$; weight: $77.1 \pm$ $9.9 \mathrm{~kg}$; BMI: $24.0 \pm 2.8 \mathrm{~kg} / \mathrm{m}^{2}$ ) were selected to participate in this study. The data were recorded during the first and second Joint European Partial-g parabolic flight (JEPPF) campaign, taking place in June 2011 and December 2012 (Pletser et al, 2012). Eight of them participated in previous partial-g or zero-g parabolic flights (experienced flyers), while the remaining six subjects experienced reduced gravity for the first time.

All subjects provided written informed consent prior to participation in the study. Each participant underwent a special flight medico-physical examination in order to pass FAA III tests. All subjects were free of any cardiopulmonary or other systemic disease. None of them were taking any medication, nor were they allowed to take either general medication or medication for the control of motion sickness before and during the parabolic flight sessions in order to eliminate the effects of pharmacological agents on cardiovascular control. Data of one subject were excluded due to severe nausea, and intake of scopolamine during the flight. The study was approved by the 'Comité Consultatif de Protection des Personnes dans la Recherche Biomédicale', the Ethical Committee of the Faculty of Medicine, KU Leuven, Belgium and the ESA medical board.

\subsection{Instrumentation}

Data of the subjects were collected using a Nexfin monitor (BMEYE, Amsterdam, The Netherlands). Pre-gelled $\mathrm{Ag} / \mathrm{AgCl}$ electrodes (Red Dot, 3M, Saint Paul, MN, USA) were pasted on the thorax for electrocardiogram (ECG) recordings. Continuous blood pressure (BP) measurements were obtained using an inflatable finger cuff with infrared plethysmography (Finapres Medical Systems, Amsterdam, The Netherlands). ECG and BP recordings were sampled at $1000 \mathrm{~Hz}$ and $200 \mathrm{~Hz}$ respectively. Accceleration data were also recorded and matched with the acceleration signal provided by ESA.

\subsection{Experimental Protocol}

The subjects participated in a parabolic flight during which micro-, lunar $(0.16 g)$ and martian $(0.38 g)$ gravity 
conditions were obtained. The flights were jointly organized by ESA, DLR, CNES and Novespace in Bordeaux, France (Pletser et al, 2012). An Airbus A300 followed a parabolic trajectory, yielding a gravity profile as shown in the top traces of Figs. 1 and 2 Each flight consisted of 31 parabolas. Between two parabolas, there was a break of almost 2 minutes. After every six parabolas, a longer break of 5 minutes was held. The data of the first parabola were never used, such that the subjects could become accustomed to the partial gravity experience. Throughout the whole flight, the subjects were seated and attached with belts around the chest to prevent free floating, and were instructed not to talk during the parabolas. In the first JEPPF campaign, there were first 12 parabolas of lunar gravity, then 12 of martian gravity, and lastly 6 parabolas that achieved microgravity conditions. During the second JEPPF campaign, the order of presentation of lunar and martian gravity was reversed.

\subsection{Data Segmentation}

Each parabola is divided in five phases, as shown in the top traces of Figs. 1 and 2 1. pre-normogravity $(1 \mathrm{~g}), 2$. pre-hypergravity $(1.7-1.8 g), 3$. reduced gravity $(0 g, 0.16$ $g$ or $0.38 g), 4$. post-hypergravity $(1.6-1.8 g)$, and 5. postnormogravity $(1 \mathrm{~g})$. The segmentation was performed according to $>1.4 \mathrm{~g}$ for pre-hypergravity, $<0.05 \mathrm{~g}$ for microgravity, $<0.20 \mathrm{~g}$ for lunar gravity, $<0.41 \mathrm{~g}$ for martian gravity and $>1.4 \mathrm{~g}$ for post-hypergravity. The pre- and post-normogravity segments were taken as the $20 \mathrm{~s}$ just before and after the parabola during which the gravity was below $1.05 \mathrm{~g}$.

\section{Methods}

The data recorded with the Nexfin monitor were converted with FrameInspector (v1.32, BMEYE), such that further processing of the data could be performed in MATLAB R2012a (MathWorks, Natick, MA, USA).

\subsection{Heart Rate Variability}

Lead II of the ECG is used to derive the variability of the heart rate. The QRS complexes are detected using the Pan-Tompkins algorithm (Pan and Tompkins, 1985) and all detections are visually verified and manually corrected if needed. The intervals between consecutive QRS complexes are the normal-to-normal (NN) interval series and constitute the tachogram.
HRV is quantified via several short-time measures, described in the Task Force on HRV (Task Force of the European Society of Cardiology and the North American Society of Pacing and Electrophysiology, 1996) in (Beckers et al, 2003: Verheyden et al, 2005), as we only have segments of 20-30 $s$ during the parabolic flights:

- Time domain HRV analysis:

- meanNN [ms]: mean of NN interval series. Its reciprocal multiplied with $60 s$ constitutes the mean heart rate;

- SDNN [ms]: standard deviation of NN interval series. SDNN is a measure of total variability;

- RMSSD [ms]: square root of the mean squared differences of successive NN intervals;

- pNN50 [\%]: percentage of NN intervals that differs more than $50 \mathrm{~ms}$ from the preceding NN interval;

- SDSD [ms]: standard deviation of successive differences.

The latter three measures are estimates of short-term components of HRV and reflect parasympathetic activity.

- Frequency domain HRV analysis:

$-\mathrm{LF}_{N N}\left[\mathrm{~ms}^{2}\right]$ : power in the low frequency range [0.04-0.15 Hz]. This measure is linked to both sympathetic and parasympathetic influences;

$-\mathrm{HF}_{N N}\left[\mathrm{~ms}^{2}\right]$ : power in the high frequency range [0.15-0.40 Hz]. HF power reflects vagal activity;

- LFnorm $_{N N}$ [nu]: relative LF power to the total power (calculated as $\mathrm{LF}_{N N}+\mathrm{HF}_{N N}$ ), expressed in normalized units. This reflects sympathovagal balance;

- HFnorm $_{N N}$ [nu]: relative HF power to the total power (calculated as $\mathrm{LF}_{N N}+\mathrm{HF}_{N N}$ ), expressed in normalized units. This is a measure of vagal activity;

- LF/HF ${ }_{N N}$ [-]: ratio between LF and HF power. This measure is an index of sympathovagal balance.

In order to reliably calculate the power spectrum for the $20 \mathrm{~s}$ segments, the procedure as described in (Verheyden et al, 2005) is followed, which includes linear trend removal, tapering with a Hamming window, assessment of stationarity and zero padding. Stationarity implies that the statistical properties of a series $[x(t)]$ do not change with time. Its assessment is performed according to the following algorithm (Beauchamp, 1973): for each $[x(t)]$, the total standard deviation $\left[S D_{t o t}\right]$ is obtained. Next, a running mean $\left\langle x_{i}\right\rangle$ is computed for all $i$ from a sliding window of six points. Finally, the stationarity requirement is met 
if $\left|<x_{i}>-<x_{i+1}>\right|<\left[S D_{t o t}\right]$. Failure to meet the stationarity requirement, lead to the removal of those segments in the calculation of spectral features.

The reliability of the computation of HRV features in these ultrashort data segments, is proven in Appendix 1.

\subsection{Blood Pressure Variability}

A systogram and a diastogram, consisting of consecutive systolic and diastolic blood pressure values respectively, are derived from the continuous blood pressure recordings. Recalibration periods of the finger plethysmography are taken into account by cubic spline interpolation that is performed using the time information derived from the QRS complexes.

The short-time BPV measures include (Laitinen et al, 1999, Parati et al, 2013, 1995):

- Time domain BPV analysis:

- meanBP [mmHg]: mean of blood pressure series;

- SDBP [mmHg]: standard deviation of blood pressure series.

- Frequency domain BPV analysis:

- $\mathrm{LF}_{B P}\left[\mathrm{mmHg}^{2}\right]$ : power in the low frequency range [0.04-0.15 Hz]. This measure is related to sympathetic vasomotor tone and sympathovagal balance;

- $\mathrm{HF}_{B P}\left[\mathrm{mmHg}^{2}\right]$ : power in the high frequency range [0.15-0.40 Hz]. This measure is linked to mechanical effects of respiration;

- LF/HF $B P$ [-]: ratio between LF and HF power. This ratio reflects the sympathovagal balance.

The same procedure as in the frequency domain HRV analysis is applied to compute the power spectrum of the systogram and diastogram.

Note that the abbreviation BP will be used when it concerns both the systogram (SBP) and diastogram (DBP).

\subsection{Statistical Analysis}

Analysis of covariance is conducted to compute the correlation coefficients with repeated observations (Bland and Altman, 1995), between the mean gravity in a certain phase, and its corresponding HRV or BPV feature, taking into account that repeated measures occur within a subject. A model with parallel lines between subjects is used. Statistical significance is considered when $p<0.05$.
Table 1: Mean durations of the different gravity phases

\begin{tabular}{r|ccc}
\hline Gravity level & phase 2 & phase 3 & phase 4 \\
\hline $0 g$ & $20.66 \pm 0.83$ & $20.38 \pm 1.05$ & $22.19 \pm 2.76$ \\
$0.16 g$ & $19.23 \pm 0.79$ & $24.60 \pm 0.64$ & $21.89 \pm 2.42$ \\
$0.38 g$ & $17.65 \pm 0.67$ & $31.37 \pm 1.06$ & $19.30 \pm 1.52$
\end{tabular}

Mean $(s) \pm$ standard deviation (s) of the duration of phases 2, 3 and 4 when zero, lunar and martian gravity levels are simulated.

\section{Results}

Table 1 displays the mean durations of the different phases when zero, lunar and martian gravity levels are obtained via parabolic flight. During the weightlessness parabolas, the hyper- and zero-gravity phases all last around $20 \mathrm{~s}$. Simulations of lunar and martian gravity result in slightly shorter hypergravity phases, but lead to longer phases of reduced gravity up to $34 s$ of martian gravity. Because the data length could influence HRV and BPV measures, we have compared time and frequency domain HRV measures in a separate experiment in 10 subjects using 20, 25 and $30 s$ segments. Differences in HRV measures related to differences in segment length were determined via the Friedman test. Except for the LF power $(p=0.045)$, none of the HRV measures presented significant differences between 20, 25 and $30 s$ segments. We, therefore, chose to use the full segments in all calculations, despite the difference in length up to $10 \mathrm{~s}$, such that all processes, present in the reduced gravity phase, will certainly be captured. Additionally, the observations of $\mathrm{LF}_{N N}$ and $\mathrm{LF}_{B P}$ will be interpreted with care.

\subsection{Dynamic Cardiovascular Response}

Figs. 1 and 2 show the time series of the $\mathrm{NN}$ interval series, systolic and diastolic blood pressures during zero and martian gravity parabolas. The time series are first averaged per subject. The mean and standard deviation of these subject-averaged time series are displayed.

From Fig. 1. we can observe that during the prehypergravity phase, the NN intervals are on average reduced up to $10 \%$ compared to the preceding normogravity phase, while both systolic and diastolic blood pressures slightly increase with respectively $5 \%$ and $10 \%$. At the onset of weightlessness, a fast increase in NN intervals of $8 \%$ compared to normogravity is observed for a few seconds, after which the NN intervals gradually decrease. During weightlessness, there is first a small, sudden increase in blood pressure, followed by a progressive decrease in SBP and DBP of respectively $15 \%$ and $20 \%$ compared to nor- 

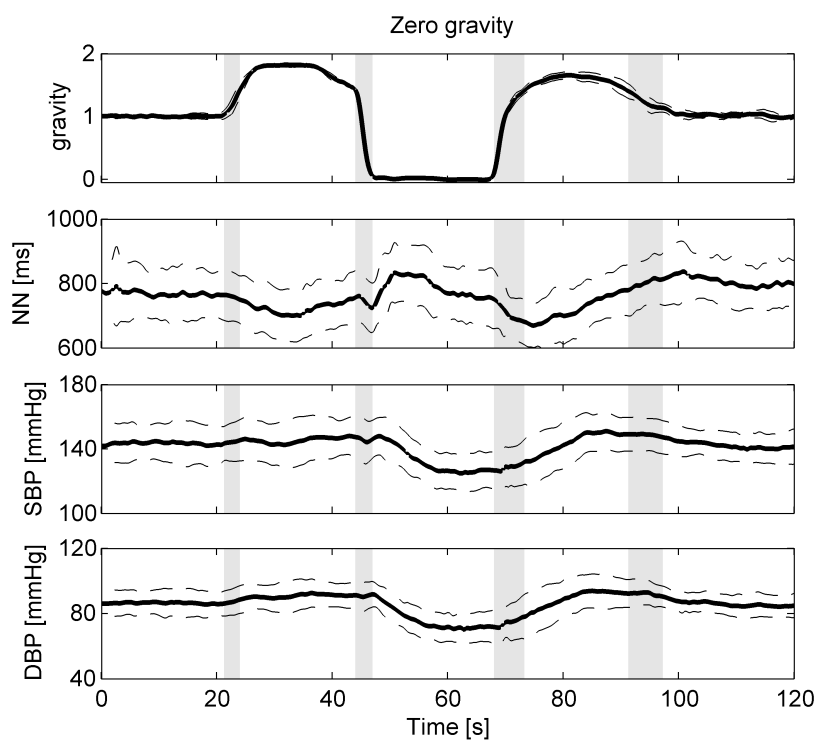

Fig. 1: Time series of NN interval series, systolic (SBP) and diastolic (DBP) blood pressure series during different gravity phases of parabolas during which zero gravity is obtained. The time series are first averaged per subject. The mean (thick line) and standard deviation (dashed lines) over all subjects are displayed. Grey areas indicate transition periods between gravity phases.

mogravity. The post-hypergravity is marked by initially low NN intervals (15\% less than normogravity) that progressively increase, on average, from $670 \mathrm{~ms}$ to $870 \mathrm{~ms}$. Both SBP and DBP present an increase during the first $10 \mathrm{~s}$ of the post-hypergravity phase from $132 \mathrm{mmHg}$ to $150 \mathrm{mmHg}$, and $80 \mathrm{mmHg}$ to $94 \mathrm{mmHg}$ respectively, after which the values stagnate at values that are on average $5 \%$ and $10 \%$ higher than during normogravity.

The dynamic cardiovascular response to a parabola during which lunar gravity was obtained (not shown), has a very similar profile as to a zero gravity parabola.

Fig. 2 displays the dynamic response during a martian gravity parabola. The response to the pre-hypergravity phase is similar as the pre-hypergravity phases during zero and lunar parabolas. At the onset of martian gravity, there is a sudden increase of $6 \%$ in NN intervals and a small increase in blood pressure, but not as much as during weightlessness. Then, the NN intervals slowly decrease, and after $10 s$ there is a stable period during which the NN intervals are still 3\% larger than during normo-gravity. At the same time, the blood pressure decreases with respectively $10 \%$ and $15 \%$ for SBP and DBP compared to normogravity during the first $10 \mathrm{~s}$ of experiencing martian gravity. Next, BP slowly increases during
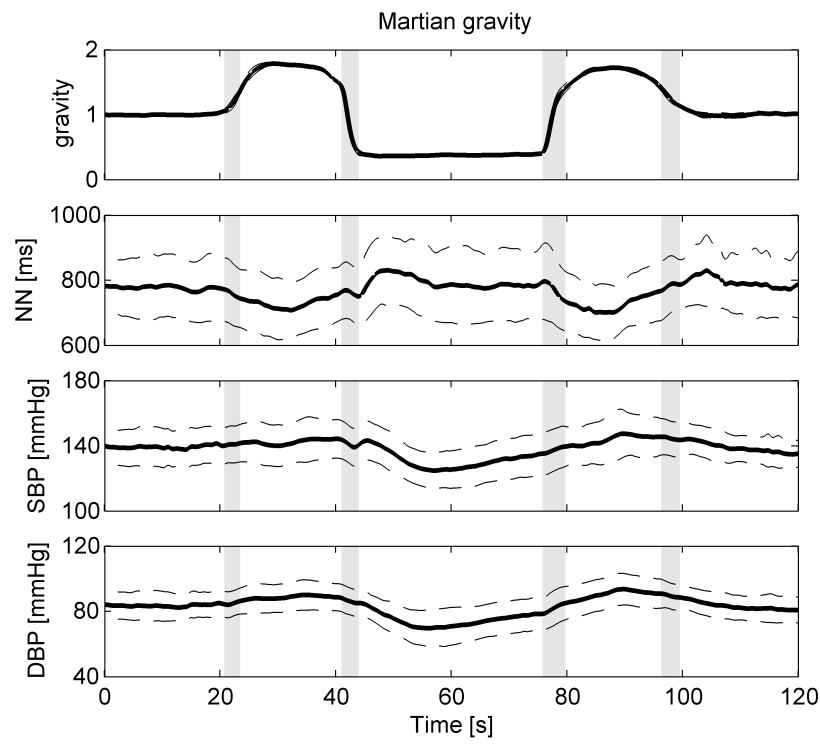

Fig. 2: Time series of NN interval series, systolic (SBP) and diastolic (DBP) blood pressure series during different gravity phases of parabolas during which martian gravity is obtained. The time series are first averaged per subject. The mean (thick line) and standard deviation (dashed lines) over all subjects are displayed. Grey areas indicate transition periods between gravity phases.

the remainder of the martian gravity phase, yet always having up to $5 \%$ and $10 \%$ lower values than during normogravity for SBP and DBP. In the post-hypergravity phase, there is first a decrease of $10 \%$ compared to normogravity in NN intervals, accompanied by an increase of 7\% in SBP and $12 \%$ in DBP compared to normogravity. In the middle of the post-hypergravity phase, the opposite response occurs.

\subsection{HRV and BPV Measures in Function of Gravity}

Tables 2 and 3 present the correlation coefficients between the HRV and BPV measures on the one hand, and the mean gravity level in each phase on the other hand. In Table 2, we can see that all temporal HRV measures have significant and negative correlation coefficients with gravity. This indicates that the variability of the heart rate is largest during weightlessness and decreases when gravity increases. From the frequency HRV measures, only $\mathrm{LF}_{N N}$ and $\mathrm{HF}_{N N}$ show significant correlations with gravity. Also here, the correlations are negative.

In Table 3, the temporal BPV measures also show significant correlations with gravity. MeanBP and $\mathrm{HF}_{S B P}$ 
Table 2: Correlation coefficients $\rho$ and slopes between the HRV measures and the gravity level $g$

\begin{tabular}{l|cr}
\hline HRV measure & $\rho$ & slope \\
\hline meanNN [ms] & $-0.36^{*}$ & -47.04 \\
SDNN [ms] & $-0.29 *$ & -10.41 \\
RMSSD [ms] & $-0.33^{*}$ & -9.01 \\
pNN50 [\%] & $-0.26^{*}$ & -4.47 \\
SDSD [ms] & $-0.32 *$ & -7.35 \\
LF $_{N N}\left[\mathrm{~ms}^{2}\right]$ & $-0.18^{*}$ & -30.98 \\
HF $_{N N}\left[\mathrm{~ms}^{2}\right]$ & $-0.18^{*}$ & -13.73 \\
LFnorm $_{N N}[\mathrm{nu}]$ & -0.08 & -0.03 \\
HFnorm $_{N N}[\mathrm{nu}]$ & 0.08 & 0.03 \\
LF/HF $_{N N}[-]$ & -0.01 & -0.14
\end{tabular}

$\rho=$ correlation coefficient; slope of HRV measure in function of $g$ (in $[H R V$ unit $/ g])$. * indicates a significant correlation.

Table 3: Correlation coefficients $\rho$ and slopes between the BPV measures and the gravity level $g$

\begin{tabular}{l|cr|cr}
\hline BPV measure & \multicolumn{2}{|c|}{ SBP } & \multicolumn{2}{c}{ DBP } \\
& \multicolumn{1}{|c}{$\rho$} & slope & $\rho$ & slope \\
\hline meanBP $[\mathrm{mmHg}]$ & $0.44^{*}$ & 8.74 & $0.67 *$ & 10.71 \\
SDBP $[\mathrm{mmHg}]$ & $-0.16^{*}$ & -0.80 & $-0.26 *$ & -0.97 \\
$\mathrm{LF}_{B P}\left[\mathrm{mmHg}^{2}\right]$ & -0.01 & -0.02 & -0.07 & -0.09 \\
$\mathrm{HF}_{B P}\left[\mathrm{mmHg}^{2}\right]$ & $0.15^{*}$ & 0.12 & 0.01 & 0.01 \\
$\mathrm{LF}_{\mathrm{H} \mathrm{HF}_{B P}[-]}$ & $-0.27^{*}$ & -9.95 & $-0.24 *$ & -9.86
\end{tabular}

$\rho=$ correlation coefficient; slope of BPV measure in function of $g$ (in $[\mathrm{BPV}$ unit $/ g]) .{ }^{*}$ indicates a significant correlation.

increase with increasing gravity, while SDBP is negatively correlated with $g$. Also $\mathrm{LF} / \mathrm{HF}_{B P}$ shows a negative relation with gravity.

In addition, several significant HRV measures are displayed in function of gravity in Fig. 3. In these plots, the difference in offset between subjects is removed, such that the within-subject trend with gravity can clearly be seen. We can observe an almost linear relation between SDNN and gravity, and a monotonic trend between RMSSD, and the gravity level. SDSD and pNN50 have a similar profile as RMSSD, but are not shown here. MeanNN also presents an increase during reduced gravity, though there is almost no difference between zero, lunar and martian gravity. On the other hand, hypergravity is related with a clear reduction in meanNN. Also $\mathrm{HF}_{N N}$ shows a decreasing trend with gravity; only during lunar gravity, an apparent drop is noticed compared to zero and martian gravity. An overview of all HRV measures in function of gravity is given in Appendix 2 in Table 6

Fig. 4 presents the profiles of several BPV measures in function of gravity, obtained in a similar way as the plots in Fig. 3. Both meanSBP and meanDBP are strongly reduced during zero and partial gravity, with again no difference between the three reduced gravity levels, while hypergravity is associated with an increase in meanBP. $\mathrm{LF} / \mathrm{HF}_{S B P}$ displays, similarly as $\mathrm{HF}_{N N}$, a decreasing value with gravity and a divergent behaviour during lunar gravity. Finally, SDDBP is also negatively correlated with gravity, with a small deflection at $1.6 \mathrm{~g}$. An overview of all BPV measures in function of gravity is given in Appendix 2 in Tables 7 and 8 .

\section{Discussion}

The aim of this study was to investigate how the autonomic adaptation of the cardiovascular system occurs during parabolic flight in which not only weightlessness was simulated, but also partial gravity levels as on the Moon and on Mars. In order to characterize cardiovascular functioning, we continuously recorded ECG and blood pressure during parabolic flight in sitting position. From the ECG and blood pressure measurements, we computed the tachogram, systogram and diastogram, from which several standardized HRV and BPV measures were derived. These indices were then used to evaluate whether a (linear) relation exists with the gravity level.

\subsection{Impact of Reduced Gravity on Cardiovascular Autonomic Control}

The effect of weightlessness induced by parabolic flight has been studied before. Iwase et al. 1999 evaluated the muscle sympathetic nerve activity (MSNA) via microneurography and found a biphasic response to weightlessness: at the onset, the thoracic fluid volume increases due to the shift of body fluids thereby reducing the mean blood pressure and suppressing MSNA, lasting around $10 \mathrm{~s}$. Afterwards, an activation of MSNA occurs. Similarly, an initial increase in blood pressure and thoracic blood volume when subjects were in standing position was noted, thereby stimulating the cardiopulmonary volume receptors and arterial baroreceptors to result in a fast bradycardia response, after which a gradual increase of the heart rate follows (Beckers et al, 2003; Liu et al, 2011; Mukai et al. 1991). During the microgravity parabolas (Fig. 1), a similar biphasic response was noted, though the initial increase in blood pressure at the onset of weightlessness is not so pronounced here. A possible explanation could be the difference in posture; in sitting position, the blood redistribution is not as large as in the standing posture. At the same time, this could also explain why the increase in NN intervals is not as large as noted by Liu et al. 2011. 

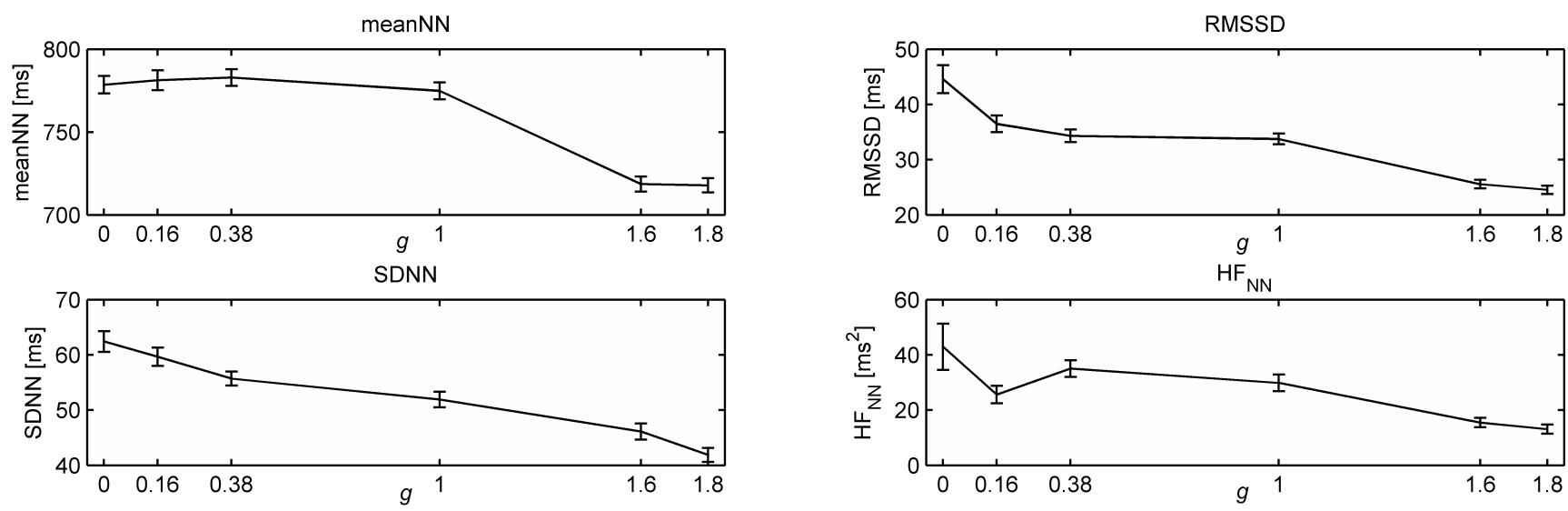

Fig. 3: HRV measures (meanNN, SDNN, RMSSD and $\mathrm{HF}_{N N}$ ) in function of the gravity level. Mean and standard errors, after removal of between-subject differences, are displayed.
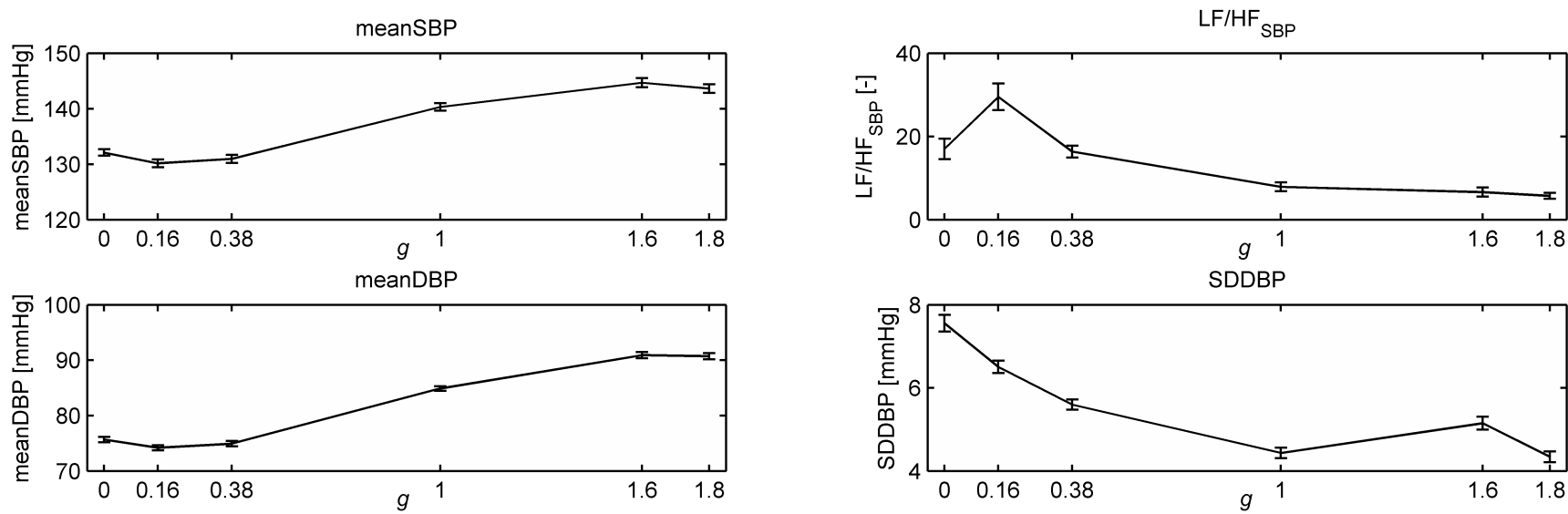

Fig. 4: BPV measures (meanSBP, meanDBP, SDDBP and $\mathrm{LF} / \mathrm{HF}_{S B P}$ ) in function of the gravity level. Mean and standard errors, after removal of between-subject differences, are displayed.

During the reduced gravity phase of a martian gravity parabola, we observed a triphasic autonomic response of the cardiovascular system (Fig. 2); at first, there is, similarly as in micro- and lunar gravity, an increase in SBP, and to a lesser extent in DBP. Because the difference between the preceding hypergravity and the martian gravity is not as large as in zero and lunar parabolas, the shift in body fluids might not be that pronounced, thereby only slightly increasing the blood pressure. Via the baroreflex, the vagal branch of the ANS is stimulated, leading to a sudden increase in NN intervals. In the second stage, we observed decreases both in blood pressure and NN intervals, up to $10 s$ after the onset of martian gravity. In the final stage, the NN intervals have reached a stable value, while the blood pressure slowly increases until the end of the martian gravity phase. The presence of this third stage could possibly be caused by one or a combination of the following factors: (1) the martian gravity period lasts on average 6 up to $11 s$ longer than the reduced gravity periods of zero and lunar gravity, allowing the NN intervals to stabilize; (2) this stage might take place within the reduced gravity period because the difference between the preceding hypergravity phase is not that large; and (3) the ratio between the proportion of cardiac output sent toward the brain and toward both the splanchnic and lower limb area could be influenced by the duration of Moon and Mars gravity period (Arbeille et al, 2008).

In addition, we have computed HRV and BPV measures to investigate whether these indices of cardiovascular autonomic control are correlated to the gravity level. As hypothesized, the results indicate negative correlations for all temporal HRV measures and few spectral HRV 
measures. The general variability of the heart rate, as indicated by SDNN, decreases linearly with increasing gravity; indeed, we can observe from Figs. 1 and 2 that there is more variability during weightlessness than during martian gravity, where in the latter, we have a relatively long stable third period in phase 3 of the parabolas. Also, all vagal indices (RMSSD, pNN50, SDSD, and $\mathrm{HF}_{N N}$ ) present a monotonic decreasing trend with gravity. As seen in the dynamic response to reduced gravity, this activation of the vagal branch probably originates from the rise in blood pressure, thereby activating the baroreflex. This rise in $\mathrm{BP}$ depends on the extent to which fluids redistribute over the body, and is largest during weightlessness. Fig. 3 also shows that there is a large difference between zero gravity and the partial gravity levels, where during the latter, vagal indices seem to be more similar to those obtained during normogravity. This indicates that the increase in vagal modulation during weightlessness is not in proportion to the reduction in gravity. The change in $\mathrm{BP}$ also affects the meanNN, though we do not observe a monotonic trend; meanNN is significantly increased during reduced gravity compared to normogravity, but there is no difference between zero, lunar and martian gravity.

A similar observation can be made for the meanBP; all reduced gravity phases have a similar meanBP, with even slightly higher values during weightlessness. This could possibly be due to the larger increase in $\mathrm{BP}$ at the onset of zero gravity before BP decreases. These larger variations also explain the decreasing trend of SDBP with increasing gravity.

Our results are comparable to those obtained during a parabolic flight organized by NASA, which replicated the gravity of the Moon and Mars; Summers et al. 2010 have reported a linear relation between the gravity level and cardiac parameters, though their parameters under study were cardiac dimensions on the left ventricular sphericity that was assessed with echocardiography.

These findings on the response of the cardiovascular autonomic control during partial gravity are important in view of future missions to the Moon and Mars. The response to prolonged weightlessness has been studied extensively and several countermeasures have been developed to deal with the cardiovascular deconditioning, and the associated postflight orthostatic intolerance (Antonutto and Di Prampero, 2003). Although further research on the effects of partial gravity on the human body is still necessary, these results might help to adapt current countermeasures to these gravity conditions, needed for a prolonged stay on Mars. Additionally, it is also important to take into account that before astronauts reach Mars, they first need to travel for a long time in microgravity. Although martian gravity is only a fraction of the Earth's gravity, there is still a risk of orthostatic intolerance upon arrival at Mars. We found that the changes in vagal activation are not in proportion with the change in gravity; the vagal response to martian gravity is more similar to normogravity than zero gravity. Therefore, in order to maximize the mission's success, the astronauts should be prepared for the altered gravity, and its associated load to the human body.

\subsection{Impact of Hypergravity on Cardiovascular Autonomic Control}

During hypergravity, Linnarsson et al. 1996 reported an increase in sympathetic outflow and withdrawal of vagal activity due to unloading of the baroreceptors. This was also found by Iwase et al. 1999 and Pump et al. 1999. Also, an increase in DBP was found during hypergravity to compensate for the decrease in stroke volume (Le Rolle et al, 2008; Liu et al, 2011). Our observations confirm these previous findings; due to the shift of the blood towards the lower extremities, the blood pressure increases, while meanNN, SDNN, SDBP and vagal indices decreased compared to normogravity and partial gravity.

This counteracting cardiovascular autonomic response to hypergravity makes it an interesting countermeasure to prevent problems after space flight. This has been studied previously by Iwasaki et al. 2005, where it was shown that intermittent exposure to hypergravity might mitigate the decrease in vagal and baroreflex activity after weightlessness, simulated via head down bed rest (HDBR). However, hypergravity, as induced via lower body negative pressure, was found to enlarge the lower limb veins thereby causing subjects to fail in a post-HDBR tilt study due to the lack of vasoconstriction (Arbeille et al, 2008).

\subsection{Methodological Considerations, Limitations and Future Work}

Parabolic flights present the only ground-based opportunity to obtain real microgravity or partial gravity levels, but brings with it several limitations, such as the short duration of the reduced gravity phase, lasting only around $20 \mathrm{~s}$. Therefore, spectral measures needed a modified computation procedure as described in (Verheyden et al 2005). Although it was shown that this specific procedure 
can be used to estimate LF and HF components, the obtained values were sometimes very large due to spectral leakage of very low frequencies. In those cases, the data segments were discarded. Additionally, LF power seemed to be significantly altered by differences in segment length (see Appendix 1). Therefore, $\mathrm{LF}_{N N}$ and $\mathrm{LF}_{B P}$ should be interpreted with care. However, all computed spectral measures should be handled cautiously; $\mathrm{LF} / \mathrm{HF}_{B P}$ and $\mathrm{HF}_{N N}$ present deviations at $0.16 \mathrm{~g}$ from the expected monotonic trends with gravity. Taking into account that RMSSD, pNN50 and SDSD are highly correlated with $\mathrm{HF}_{N N}$, this seems rather a methodological issue than a change in vagal modulation.

Another limitation of parabolic flight is the presence of hypergravity phases. We can therefore only study fastly changing mechanisms as we would otherwise study the response to the preceding hypergravity phase rather than the response to reduced gravity. However, the findings on cardiovascular autonomic control during parabolic flight correspond to those in space. Yet, in the dynamic response during parabolic flight, we can identify a bior triphasic response within the reduced gravity phase, possibly giving rise to nonstationarities. Therefore, in future studies, we aim to conduct time-frequency analyses to trace the spectral measures in time; these analyses combine the advantages of time and frequency analyses and can be used for nonstationary signals (Orini et al. 2012).

Beckers et al. 2003 reported large differences in response in subjects experiencing two parabolic flights; during the first flight, the response to the first ten parabolas were significantly different compared to the first parabolas of the second flight. Only after ten parabolas, the subjects were not stressed or excited anymore, therefore they only studied the data originating from the second flight. Unfortunately, we were not able to submit all of our subjects to multiple flights, which could result in increased sympathetic responses during reduced gravity in the first parabolas. By reversing the presentation order of lunar and martian gravity in the first and second partial-g campaigns, the impact of this effect is equally spread over the partial gravity levels. Due to the limited number of parabolas in different gravity levels, we were also not able to use only the last 15 or 20 parabolas.

Additionally, Beckers et al. 2003 also suggested that experienced parabolic flyers seem to have a different response during microgravity than first time flyers, possibly due to their active change in breathing pattern, thereby changing the vagal response (Iwase et al, 1998). We have visually compared the response of the experienced and first time flyers but could not find any difference. It could be that the breathing was controlled in various ways by experienced flyers, causing different autonomic responses. Therefore, respiration should be studied simultaneously and taken into account in HRV analyses as suggested in (Widjaja et al, 2014). Note, however, that it was initially the intention to conduct a joint analysis of the respiratory and cardiovascular systems. However, the recordings of respiration during the first campaign were unreliable due to technical problems, and thus surrogate respiratory signals, such as ECG-derived respiration (Widjaja et al, 2012), should be computed for future analyses.

A last remark concerns the motion sickness of which several subjects suffered from because they were not allowed to take any medication. As a consequence, one experienced flyer was severely nauseated and received medication during the parabolic flight, and hence was discarded from the analyses. Other subjects, experienced and not, were also nauseated for a short time, during which their data were also excluded, but they recovered completely and they could proceed with the recordings without any medication. Nausea and vomiting activate several sympathetic and vagal responses, and thus might interfere with the autonomic response to changing gravity. We, however, noted no differences in response in nauseated subjects.

\section{Conclusion}

In this paper, the response of the cardiovascular system to partial gravity levels, as on the Moon and on Mars, was studied. A biphasic response to zero and lunar gravity, induced by parabolic flight, was noted, while martian gravity presented a triphasic response. Additionally, significant correlations were observed between the gravity level and several measures of heart rate and blood pressure variability, indicating that (1) the heart rate, the reciprocal of meanNN, and blood pressure increase; and (2) vagal activity decreases, with increasing gravity.

\section{Acknowledgements}

We thank the subjects who volunteered for this study. We acknowledge the support from the European Space Agency and Novespace and the collaboration of the crew of the Airbus A300 ZERO-G, and a special thanks to Mr. Vladimir Pletser (ESA). 
Research supported by Research Council KUL: CoE PFV /10/002 (OPTEC), PhD/Postdoc grants; IWT: TBM 080658-MRI (EEG-fMRI), TBM 110697-NeoGuard, PhD/ Postdoc grants, D. Widjaja is supported by an IWT PhD Grant and the Zonta Amelia Earhart Fellowship 2014-2015; FWO: G.0427.10N (Integrated EEG-fMRI), G.0869.12N (Tumor imaging), G.0108.11 (Compressed Sensing), G.0A5513N (Deep brain stimulation), PhD/Postdoc grants; iMinds Medical Information Technologies: ICON NXT_Sleep, SBO 2015; Flanders Care Demonstratieproject Tele-Rehab III (2012-2014); ESA AO-PGPF-01, PRODEX (CardioControl) C4000103224; Belgian Federal Science Policy Office IUAP P7/19 (DYSCO, 'Dynamical systems, control and optimization', 2012-2017); Belgian Foreign Affairs-Development Cooperation: VLIR UOS programs; EU RECAP 209G within INTERREG IVB NWE programme, EU MC ITN TRANSACT 2012 (no 316679), ERASMUS EQR Community service engineer (no 539642-LLP-1-2013); EU: the research leading to these results has received funding from the European Research Council under the European Union's Seventh Framework Programme (FP7/20072013)/ERC Advanced Grant: BIOTENSORS (no 339804). This paper reflects only the authors' views and the Union is not liable for any use that may be made of the contained information.

\section{Appendix 1: HRV analysis of ultrashort data segments}

\section{A1.1 Power spectral analysis of ultrashort data segments}

This proof is reproduced from (Verheyden et al, 2005) with permission from the editor.

The influence of initial signal processing on the resulting power spectral density (PSD: $\mathrm{ms}^{2} / \mathrm{Hz}$ ) function of ultrashort data segments is represented by a simulated RR tachogram (Fig. 5) and a physiological correlate (Fig. 6). Fig. 5 demonstrates a time series, created by the sum of two sinusoidal waveforms of 0.1 and $0.3 \mathrm{~Hz}$ and equal amplitude. A tendency of RR to increase from $\pm 800 \mathrm{~ms}$ in the beginning towards $\pm 950 \mathrm{~ms}$ after $20 \mathrm{~s}$ can be observed. At the left side of Fig. 5, the original time series is shown together with its PSD function. The right side shows the same signals in time and frequency domain after DC and trend removal, applying a Hamming window function and zero padding. It is obvious that with this procedure the proportion of spectral components is better preserved. Due to the artificially enhanced frequency resolution, the variance of each FFT epoch is reduced, spectral leakage is suppressed and selectivity is improved. At the same time, it causes smoothing of the power spectrum.

Fig. 6 shows the modified spectral procedure in a representative RR series obtained from a standing subject at phase 1 of parabolic flight. The distribution of powers is characterized by two frequency components around 0.1 and $0.3 \mathrm{~Hz}$, similar to short-term heart rate recordings of several minutes (Malik and Camm, 1993). The lowest measurable cyclic component remains limited due to the length of the data recording; i.e., a time window of, e.g., $\pm 20 \mathrm{~s}(\mathrm{~T})$. This allows a theoretical lower limit oscillatory frequency of $\pm 0.05 \mathrm{~Hz}(1 / \mathrm{T})$. However, practically it is advised to have at least two oscillations within the time window (Beauchamp, 1973).

\section{A1.2 HRV analysis of ultrashort data segments}

The use and validation of short-term HRV analyses has been thoroughly discussed in (Hartikainen et al, 1998). Additionally, experiments were conducted with real data of 10 male subjects (age: 25-29 yr) that were in resting supine and standing position, each for 5 minutes.

In the first experiment, postural differences in both time and frequency domain HRV measures were compared, when the first $25 \mathrm{~s}$ of the recordings were used, and when the full recordings $(5 \mathrm{~min})$ were used. Table 4 presents the first, second and third quartiles of the HRV measures in supine and standing posture. Differences in posture were assessed via the Wilcoxon signed rank test. When comparing the time domain HRV measures of $25 \mathrm{~s}$ and $5 \mathrm{~min}$, we can observe that they both have the same order of magnitude. Postural differences are observed in all cases, whether the first $25 \mathrm{~s}$ are used for the computation of time domain HRV measures, or the full recording. In the frequency domain, as expected, the LF and HF power differ a few orders of magnitudes due to the difference in record length. However, the observations in postural differences are similar in both record lengths, except for LF/HF. The ratio between both powers seems to increase in standing posture. This is yet only significant in the 5 min segment. It is thus possible that during the analyses of the PFC, differences in LF/HF might not be captured in the ultrashort data segments.

In a second experiment, we aimed to assess whether segment lengths of 20, 25 and 30 s have different HRV measures. To this end, data of the first 20, 25 and 30 in supine posture were used to compute the HRV measures. Table 5 presents the first, second and third quartiles of the HRV measures in supine posture for the different segment 

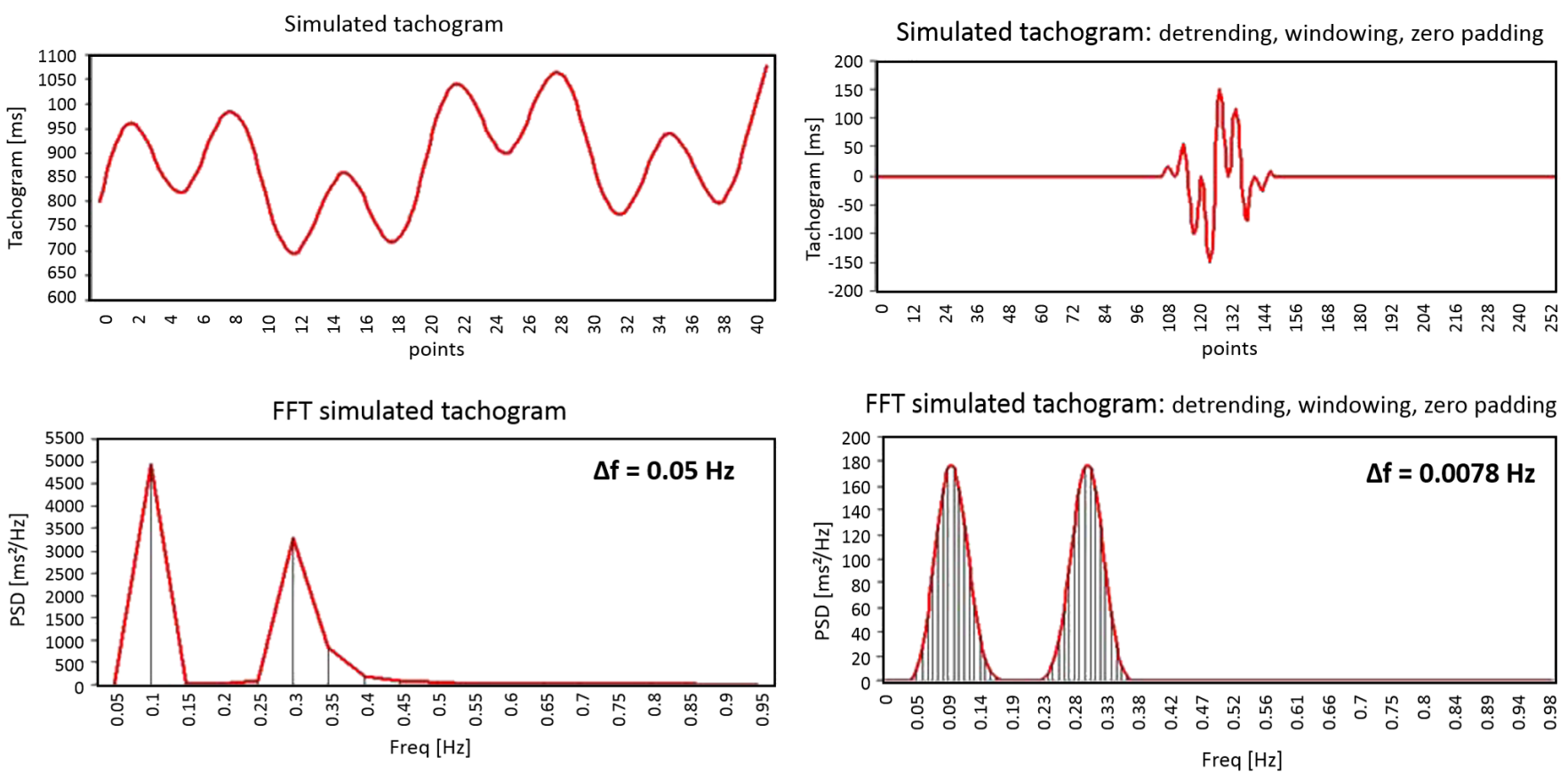

FFT simulated tachogram: detrending, windowing, zero padding

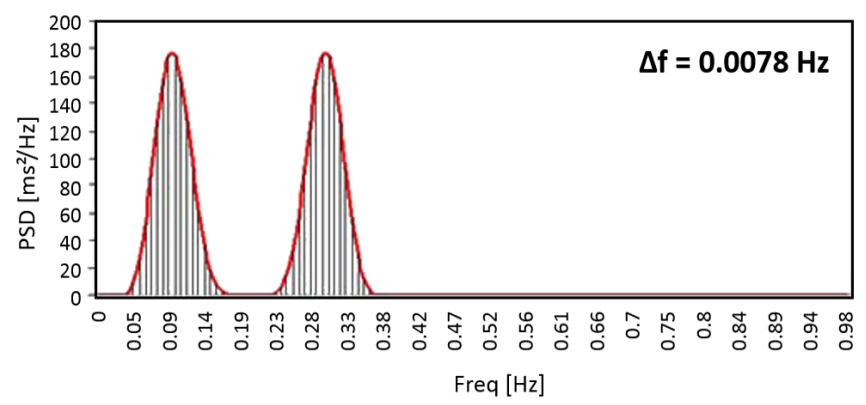

Fig. 5: Simulated RR oscillation.
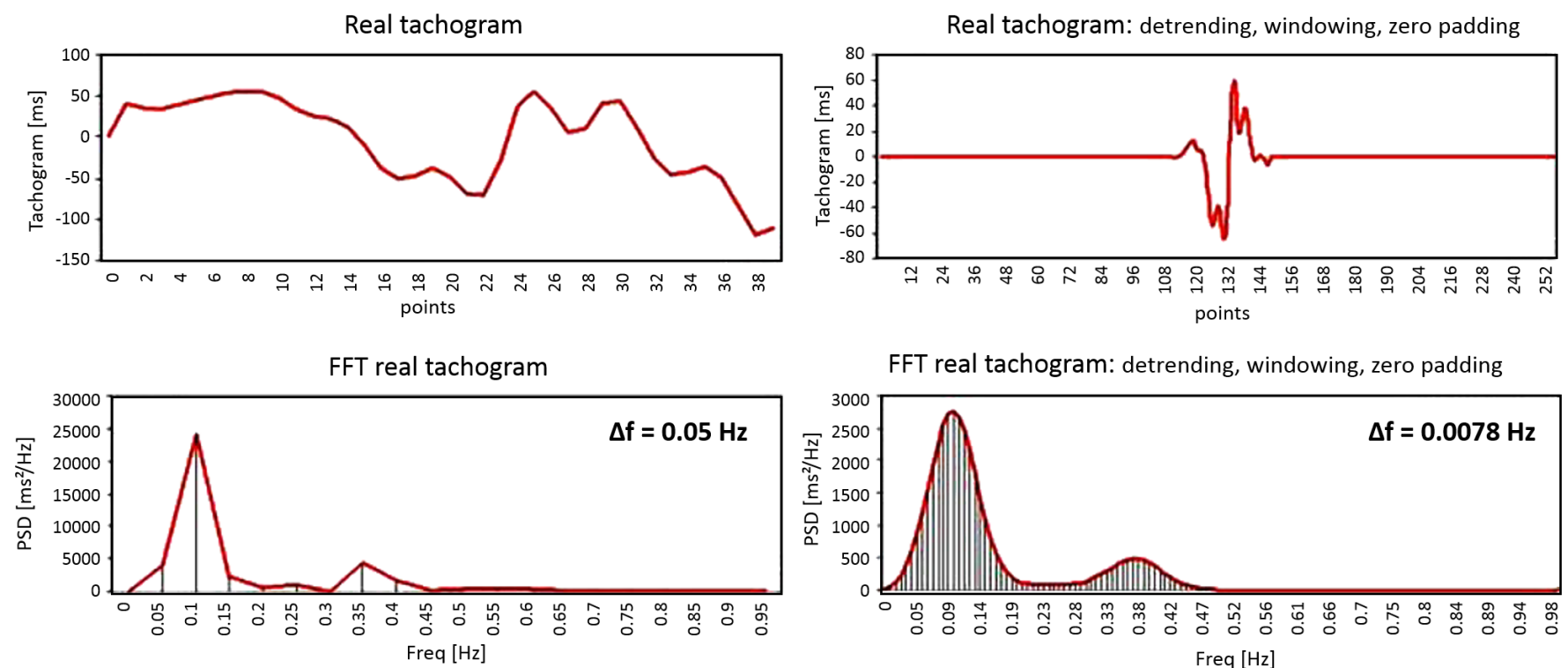

FFT real tachogram: detrending, windowing, zero padding

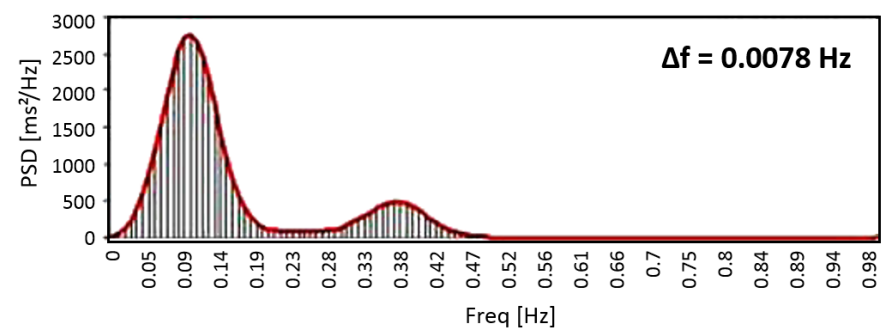

Fig. 6: Real tachogram.

lengths. Differences between the segment lengths are assessed via the Friedman test. This reveals that, except for LF, none of the HRV measures differ significantly between the segment lengths. Only LF presents a significant effect of the difference in segment length, with a significant difference between $20 \mathrm{~s}$ and $30 \mathrm{~s}$. This shows that in this study, LF power should be interpreted with care, both in HRV as BPV analyses, as changes in LF with gravity might be caused by differences in segment length.

The results from these experiments confirm that differences in autonomic control can reliably be observed, even in ultrashort recordings, for all HRV and BPV measures. Only the LF power should be interpreted with care. 
Table 4: First, second and third quartiles of HRV measures of 10 subjects in supine and standing posture, calculated using the first $25 \mathrm{~s}$ or the full $5 \mathrm{~min}$ recording.

\begin{tabular}{|c|c|c|c|c|c|c|c|}
\hline & & \multicolumn{3}{|c|}{$25 \mathrm{~s}$} & \multicolumn{3}{|c|}{$5 \mathrm{~min}$} \\
\hline & & supine & standing & $p$-value & supine & standing & $p$-value \\
\hline \multirow{3}{*}{ meanNN [ms] } & $25 \%$ & 788.00 & 720.89 & \multirow{3}{*}{$<0.01$} & 785.46 & 672.01 & \multirow{3}{*}{$<0.01$} \\
\hline & $50 \%$ & 852.41 & 759.00 & & 839.38 & 740.29 & \\
\hline & $75 \%$ & 894.90 & 855.58 & & 918.16 & 819.49 & \\
\hline \multirow{3}{*}{ SDNN [ms] } & $25 \%$ & 40.02 & 28.09 & \multirow{3}{*}{$<0.05$} & 40.80 & 36.63 & \multirow{3}{*}{$<0.05$} \\
\hline & $50 \%$ & 52.51 & 38.59 & & 71.27 & 50.47 & \\
\hline & $75 \%$ & 114.68 & 58.23 & & 83.29 & 67.82 & \\
\hline \multirow{3}{*}{ RMSSD [ms] } & $25 \%$ & 44.90 & 17.93 & \multirow{3}{*}{$<0.05$} & 34.92 & 19.83 & \multirow{3}{*}{$<0.01$} \\
\hline & $50 \%$ & 53.73 & 31.44 & & 55.25 & 30.92 & \\
\hline & $75 \%$ & 116.34 & 50.92 & & 70.01 & 45.03 & \\
\hline \multirow{3}{*}{ pNN50 [\%] } & $25 \%$ & 21.21 & 0.00 & \multirow{3}{*}{$<0.01$} & 15.25 & 0.58 & \multirow{3}{*}{$<0.05$} \\
\hline & $50 \%$ & 26.38 & 11.07 & & 30.11 & 11.06 & \\
\hline & $75 \%$ & 50.00 & 38.24 & & 48.52 & 26.86 & \\
\hline \multirow{3}{*}{ SDSD [ms] } & $25 \%$ & 21.58 & 9.99 & \multirow{3}{*}{$<0.05$} & 23.52 & 11.61 & \multirow{3}{*}{$<0.05$} \\
\hline & $50 \%$ & 33.15 & 16.82 & & 32.90 & 19.26 & \\
\hline & $75 \%$ & 75.05 & 30.34 & & 44.87 & 27.68 & \\
\hline \multirow{3}{*}{$\mathrm{LF}\left[\mathrm{ms}^{2}\right]$} & $25 \%$ & 55.06 & 26.70 & \multirow{3}{*}{$<0.05$} & 592.61 & 601.35 & \multirow{3}{*}{$<0.05$} \\
\hline & $50 \%$ & 74.94 & 45.17 & & 1286.51 & 708.51 & \\
\hline & $75 \%$ & 195.53 & 138.13 & & 2003.48 & 1585.32 & \\
\hline \multirow{3}{*}{$\mathrm{HF}\left[\mathrm{ms}^{2}\right]$} & $25 \%$ & 37.43 & 11.83 & \multirow{3}{*}{$<0.05$} & 716.94 & 113.49 & \multirow{3}{*}{$<0.05$} \\
\hline & $50 \%$ & 77.57 & 30.73 & & 1048.56 & 190.76 & \\
\hline & $75 \%$ & 212.49 & 91.47 & & 1357.00 & 1233.39 & \\
\hline \multirow{3}{*}{ LFnorm $_{N N}[\mathrm{nu}]$} & $25 \%$ & 0.22 & 0.32 & \multirow{3}{*}{$<0.05$} & 0.25 & 0.36 & \multirow{3}{*}{$<0.05$} \\
\hline & $50 \%$ & 0.43 & 0.59 & & 0.53 & 0.79 & \\
\hline & $75 \%$ & 0.61 & 0.89 & & 0.83 & 0.90 & \\
\hline \multirow{3}{*}{ HFnorm $_{N N}[\mathrm{nu}]$} & $25 \%$ & 0.39 & 0.11 & \multirow{3}{*}{$<0.05$} & 0.17 & 0.10 & \multirow{3}{*}{$<0.05$} \\
\hline & $50 \%$ & 0.57 & 0.41 & & 0.47 & 0.21 & \\
\hline & $75 \%$ & 0.78 & 0.68 & & 0.75 & 0.64 & \\
\hline & $25 \%$ & 0.27 & 0.48 & & 0.33 & 0.57 & \\
\hline $\mathrm{LF} / \mathrm{HF}_{N N}[-]$ & $50 \%$ & 0.78 & 1.45 & 0.08 & 1.16 & 4.77 & $<0.05$ \\
\hline & $75 \%$ & 1.57 & 8.12 & & 4.73 & 9.18 & \\
\hline
\end{tabular}

\section{Appendix 2}

Tables 6, 7 and 8 give the mean \pm standard error for each HRV and BPV measure in function of the gravity level. The offset between subjects is removed, such that the within-subject trend with gravity can clearly be seen.

\section{References}

Aerts W, Joosen P, Widjaja D, Varon C, Vandeput S, Van Huffel S, Aubert AE (2012) Heart rate and blood pressure variability under moon, mars and zero gravity conditions during parabolic flights. In: Proc. of the Joint Life Science Meeting 'Life in Space for Life on Earth'

Antonutto G, Di Prampero PE (2003) Cardiovascular deconditioning in microgravity: some possible countermeasures. Eur J Appl Physiol 90(3-4):283-291, DOI 10.1007/s00421-003-0884-5
Arbeille P, Kerbeci P, Mattar L, Shoemaker JK, Hughson R (2008) Insufficient flow reduction during lbnp in both splanchnic and lower limb areas is associated with orthostatic intolerance after bedrest. Am J Physiol Heart Circ Physiol 295(5):H1846-H1854, DOI 10.1152/ajpheart.509.2008

Aubert AE, Beckers F, Verheyden B (2005) Cardiovascular function and basics of physiology in microgravity. Acta Cardiol 60(2):129-151, DOI 10.2143/AC.60.2.2005024

Baevsky RM, Baranov VM, Funtova II, Diedrich A, Pashenko AV, Chernikova AG, Drescher J, Jordan J, Tank J (2007) Autonomic cardiovascular and respiratory control during prolonged spaceflights aboard the international space station. J Appl Physiol 103(1):156-161, DOI 10.1152/japplphysiol.00137.2007

Beauchamp KG (1973) Signal processing using analog and digital techniques. Allen \& Unwin, London

Beckers F, Seps B, Ramaekers D, Verheyden B, Aubert AE (2003) Parasympathetic heart rate modulation during 
Table 5: First, second and third quartiles of HRV measures of 10 subjects in supine posture, calculated using the first 20, 25 and $30 \mathrm{~s}$ of the recording.

\begin{tabular}{|c|c|c|c|c|c|}
\hline & & $20 \mathrm{~s}$ & $25 \mathrm{~s}$ & $30 \mathrm{~s}$ & $p$-value \\
\hline \multirow{3}{*}{ meanNN [ms] } & $25 \%$ & 787.22 & 788.00 & 787.08 & \multirow{3}{*}{0.12} \\
\hline & $50 \%$ & 848.08 & 852.41 & 859.13 & \\
\hline & $75 \%$ & 882.88 & 894.90 & 901.77 & \\
\hline \multirow{3}{*}{ SDNN [ms] } & $25 \%$ & 42.06 & 40.02 & 37.20 & \multirow{3}{*}{0.74} \\
\hline & $50 \%$ & 51.37 & 52.51 & 56.59 & \\
\hline & $75 \%$ & 108.80 & 114.68 & 116.35 & \\
\hline \multirow{3}{*}{ RMSSD [ms] } & $25 \%$ & 45.24 & 44.90 & 42.87 & \multirow{3}{*}{0.74} \\
\hline & $50 \%$ & 54.20 & 53.73 & 53.35 & \\
\hline & $75 \%$ & 111.33 & 116.34 & 115.81 & \\
\hline \multirow{3}{*}{ pNN50 [\%] } & $25 \%$ & 24.00 & 21.21 & 17.50 & \multirow{3}{*}{0.84} \\
\hline & $50 \%$ & 26.62 & 26.38 & 30.30 & \\
\hline & $75 \%$ & 50.00 & 50.00 & 48.65 & \\
\hline \multirow{3}{*}{ SDSD [ms] } & $25 \%$ & 22.49 & 21.58 & 22.01 & \multirow{3}{*}{0.15} \\
\hline & $50 \%$ & 34.34 & 33.15 & 33.56 & \\
\hline & $75 \%$ & 80.06 & 75.05 & 76.00 & \\
\hline \multirow{3}{*}{$\mathrm{LF}\left[\mathrm{ms}^{2}\right]$} & $25 \%$ & 37.58 & 55.06 & 46.76 & \multirow{3}{*}{$<0.05$} \\
\hline & $50 \%$ & 65.54 & 74.94 & 84.96 & \\
\hline & $75 \%$ & 96.04 & 195.53 & 217.81 & \\
\hline \multirow{3}{*}{$\mathrm{HF}\left[\mathrm{ms}^{2}\right]$} & $25 \%$ & 33.80 & 37.43 & 38.75 & \multirow{3}{*}{0.08} \\
\hline & $50 \%$ & 72.67 & 77.57 & 87.62 & \\
\hline & $75 \%$ & 103.31 & 212.49 & 371.26 & \\
\hline \multirow{3}{*}{$\operatorname{LFnorm}_{N N}[\mathrm{nu}]$} & $25 \%$ & 0.28 & 0.22 & 0.21 & \multirow{3}{*}{0.27} \\
\hline & $50 \%$ & 0.39 & 0.43 & 0.47 & \\
\hline & $75 \%$ & 0.66 & 0.61 & 0.56 & \\
\hline \multirow{3}{*}{ HFnorm $_{N N}[\mathrm{nu}]$} & $25 \%$ & 0.34 & 0.39 & 0.44 & \multirow{3}{*}{0.27} \\
\hline & $50 \%$ & 0.61 & 0.57 & 0.53 & \\
\hline & $75 \%$ & 0.72 & 0.78 & 0.79 & \\
\hline \multirow{3}{*}{$\mathrm{LF} / \mathrm{HF}_{N N}[-]$} & $25 \%$ & 0.38 & 0.27 & 0.26 & \multirow{3}{*}{0.27} \\
\hline & $50 \%$ & 0.66 & 0.78 & 0.90 & \\
\hline & $75 \%$ & 1.93 & 1.57 & 1.28 & \\
\hline
\end{tabular}

Table 6: HRV measures [mean \pm standard error] in function of the gravity level $g$. The offset between subjects is removed.

\begin{tabular}{l|rrrrrr}
\hline & \multicolumn{1}{|c}{$0 \mathrm{~g}$} & \multicolumn{1}{c}{$0.16 \mathrm{~g}$} & \multicolumn{1}{c}{$0.38 \mathrm{~g}$} & \multicolumn{1}{c}{$1 \mathrm{~g}$} & \multicolumn{1}{c}{$1.6 \mathrm{~g}$} & \multicolumn{1}{c}{$1.8 g$} \\
\hline meanNN [ms] & $778.56 \pm 5.23$ & $781.29 \pm 5.99$ & $782.88 \pm 4.99$ & $774.86 \pm 5.10$ & $718.63 \pm 4.54$ & $717.84 \pm 4.28$ \\
SDNN [ms] & $62.42 \pm 1.88$ & $59.65 \pm 1.66$ & $55.71 \pm 1.27$ & $51.91 \pm 1.40$ & $46.13 \pm 1.46$ & $41.89 \pm 1.26$ \\
RMSSD [ms] & $44.56 \pm 2.54$ & $36.47 \pm 1.52$ & $34.30 \pm 1.14$ & $33.75 \pm 0.97$ & $25.57 \pm 0.79$ & $24.55 \pm 0.76$ \\
pNN50 [\%] & $13.69 \pm 0.76$ & $12.20 \pm 0.78$ & $12.16 \pm 0.60$ & $13.21 \pm 0.73$ & $7.26 \pm 0.64$ & $6.20 \pm 0.57$ \\
SDSD [ms] & $33.74 \pm 2.31$ & $25.27 \pm 1.32$ & $23.24 \pm 1.02$ & $21.65 \pm 0.77$ & $16.30 \pm 0.60$ & $15.73 \pm 0.61$ \\
LF $_{N N}\left[\mathrm{~ms}^{2}\right]$ & $80.44 \pm 6.76$ & $91.32 \pm 7.46$ & $98.41 \pm 7.65$ & $90.90 \pm 7.94$ & $52.40 \pm 5.29$ & $49.62 \pm 5.00$ \\
HF $_{N N}\left[\mathrm{~ms}^{2}\right]$ & $42.94 \pm 8.39$ & $25.61 \pm 3.21$ & $35.02 \pm 3.03$ & $29.85 \pm 3.06$ & $15.49 \pm 1.69$ & $13.09 \pm 1.66$ \\
LFnorm $_{N N}[\mathrm{nu}]$ & $0.73 \pm 0.02$ & $0.77 \pm 0.01$ & $0.73 \pm 0.01$ & $0.72 \pm 0.01$ & $0.70 \pm 0.01$ & $0.72 \pm 0.02$ \\
HFnorm $_{N N}[\mathrm{nu}]$ & $0.27 \pm 0.02$ & $0.23 \pm 0.01$ & $0.27 \pm 0.01$ & $0.28 \pm 0.01$ & $0.30 \pm 0.01$ & $0.28 \pm 0.02$ \\
LF/HF $_{N N}[-]$ & $7.31 \pm 0.86$ & $8.13 \pm 0.71$ & $5.91 \pm 0.73$ & $6.12 \pm 0.65$ & $6.59 \pm 0.72$ & $7.25 \pm 0.70$ \\
\hline
\end{tabular}

parabolic flights. Eur J Appl Physiol 90(1):83-91, DOI 10.1007/s00421-003-0854-y

Bland JM, Altman DG (1995) Calculating correlation coefficients with repeated observations: Part 1-correlation within subjects. BMJ 310(6977):446, DOI 10.1136/bmj. 310.6977 .446
Buckey Jr JC, Lane LD, Levine BD, Watenpaugh DE, Wright SJ, Moore WE, Gaffney F, Blomqvist CG (1996) Orthostatic intolerance after spaceflight. J Appl Physiol (1985) 81(1):7-18

Cooke WH, Ames JE, Crossman AA, Cox JF, Kuusela TA, Tahvanainen KU, Moon LB, Drescher J, Baisch FJ, Mano $\mathrm{T}$, et al (2000) Nine months in space: effects on human 
Table 7: BPV measures of the systogram [mean \pm standard error] in function of the gravity level $g$. The offset between subjects is removed.

\begin{tabular}{|c|c|c|c|c|c|c|}
\hline & $0 g$ & $0.16 g$ & $0.38 g$ & $1 g$ & $1.6 \mathrm{~g}$ & $1.8 \mathrm{~g}$ \\
\hline meanSBP [mmHg] & $132.11 \pm 0.60$ & $130.15 \pm 0.69$ & $130.96 \pm 0.72$ & $140.31 \pm 0.67$ & $144.68 \pm 0.83$ & $143.62 \pm 0.77$ \\
\hline SDSBP [mmHg] & $9.66 \pm 0.25$ & $8.50 \pm 0.20$ & $7.27 \pm 0.17$ & $5.84 \pm 0.17$ & $7.11 \pm 0.21$ & $6.48 \pm 0.19$ \\
\hline $\mathrm{LF}_{S B P}\left[\mathrm{mmHg}^{2}\right]$ & $0.96 \pm 0.08$ & $1.25 \pm 0.08$ & $1.33 \pm 0.08$ & $1.10 \pm 0.08$ & $1.18 \pm 0.11$ & $1.15 \pm 0.10$ \\
\hline $\mathrm{HF}_{S B P}\left[\mathrm{mmHg}^{2}\right]$ & $0.28 \pm 0.05$ & $0.12 \pm 0.02$ & $0.18 \pm 0.03$ & $0.29 \pm 0.03$ & $0.34 \pm 0.04$ & $0.37 \pm 0.04$ \\
\hline $\mathrm{LF} / \mathrm{HF}_{S B P}[-]$ & $17.01 \pm 2.45$ & $29.51 \pm 3.21$ & $16.34 \pm 1.45$ & $7.89 \pm 1.06$ & $6.62 \pm 1.10$ & $5.71 \pm 0.71$ \\
\hline
\end{tabular}

Table 8: BPV measures of the diastogram [mean \pm standard error] in function of the gravity level $g$. The offset between subjects is removed.

\begin{tabular}{l|rrrrrr}
\hline & \multicolumn{1}{c}{$0 \mathrm{~g}$} & \multicolumn{1}{c}{$0.16 \mathrm{~g}$} & \multicolumn{1}{c}{$0.38 \mathrm{~g}$} & \multicolumn{1}{c}{$1 \mathrm{~g}$} & \multicolumn{1}{c}{$1.6 \mathrm{~g}$} & \multicolumn{1}{c}{$1.8 \mathrm{~g}$} \\
\hline meanDBP $[\mathrm{mmHg}]$ & $75.67 \pm 0.50$ & $74.19 \pm 0.46$ & $74.92 \pm 0.48$ & $84.89 \pm 0.41$ & $90.91 \pm 0.57$ & $90.71 \pm 0.55$ \\
SDDBP [mmHg] & $7.56 \pm 0.20$ & $6.51 \pm 0.15$ & $5.60 \pm 0.12$ & $4.43 \pm 0.13$ & $5.15 \pm 0.15$ & $4.34 \pm 0.13$ \\
$\mathrm{LF}_{D B P}\left[\mathrm{mmHg}^{2}\right]$ & $0.57 \pm 0.03$ & $0.73 \pm 0.04$ & $0.80 \pm 0.05$ & $0.62 \pm 0.05$ & $0.62 \pm 0.05$ & $0.53 \pm 0.04$ \\
$\mathrm{HF}_{D B P}\left[\mathrm{mmHg}^{2}\right]$ & $0.16 \pm 0.03$ & $0.07 \pm 0.01$ & $0.09 \pm 0.02$ & $0.14 \pm 0.02$ & $0.11 \pm 0.01$ & $0.12 \pm 0.02$ \\
$\mathrm{LF}^{2} \mathrm{HF}_{D B P}[-]$ & $21.25 \pm 2.00$ & $32.29 \pm 3.23$ & $20.58 \pm 1.62$ & $12.19 \pm 1.40$ & $10.76 \pm 1.34$ & $9.51 \pm 1.20$ \\
\hline
\end{tabular}

autonomic cardiovascular regulation. J Appl Physiol (1985) 89(3):1039-1045

Evans JM, Mohney L, Wang S, Moore RK, Elayi SC, Stenger MB, Moore FB, Knapp CF (2013) Cardiovascular regulation during body unweighting by lower body positive pressure. Aviat Space Environ Med 84(11):11401146, DOI 10.3357/ ASEM.3576.2013

Hartikainen JE, Tahvanainen KU, Kuusela TA (1998) Short-term measurement of heart rate variability. In: Malik M (ed) Clinical guide to cardiac autonomic tests, Springer, The Netherlands, pp 149-176, DOI 10.1007/978-94-017-1057-2_6

Iwasaki Ki, Shiozawa T, Kamiya A, Michikami D, Hirayanagi K, Yajima K, Iwase S, Mano T (2005) Hypergravity exercise against bed rest induced changes in cardiac autonomic control. Eur J Appl Physiol 94(3):285291, DOI 10.1007/s00421-004-1308-x

Iwase S, Mano T, Cui J, Kitazawa H, Kamiya A, Miyazaki S, Sugiyama Y, Mukai C, Kohno M, Nagaoka S (1998) Changes in muscle sympathetic nerve activity and effect of breathing maneuvers during microgravity induced by parabolic flight in humans. Environ Med 42(2):152155

Iwase S, Mano T, Cui J, Kitazawa H, Kamiya A, Miyazaki S, Sugiyama Y, Mukai C, Nagaoka S (1999) Sympathetic outflow to muscle in humans during short periods of microgravity produced by parabolic flight. Am J Physiol Regul Integr Comp Physiol 277(2):R419-R426

Kostas VI, Stenger MB, Knapp CF, Shapiro R, Wang S, Diedrich A, Evans JM (2014) Cardiovascular models of simulated moon and mars gravities: Head-up tilt vs. lower body unweighting. Aviat Space Environ Med 85(4):414-419, DOI 10.3357/ASEM.3687.2014

Laitinen T, Hartikainen J, Niskanen L, Geelen G, Länsimies E (1999) Sympathovagal balance is major determinant of short-term blood pressure variability in healthy subjects. Am J Physiol Heart Circ Physiol 276(4):H1245-H1252

Le Rolle V, Hernández AI, Richard PY, Carrault G (2008) An autonomic nervous system model applied to the analysis of orthostatic tests. Modelling and Simulation in Engineering 2008:2, DOI 10.1155/2008/427926

Linnarsson D, Sundberg CJ, Tedner B, Haruna Y, Karemaker JM, Antonutto G, Di Prampero PE (1996) Blood pressure and heart rate responses to sudden changes of gravity during exercise. Am J Physiol Heart Circ Physiol 270(6):H2132-H2142

Liu J, Verheyden B, Beckers F, Aubert AE (2011) Haemodynamic adaptation during sudden gravity transitions. Eur J Appl Physiol 112(1):79-89, DOI 10.1007/ s00421-011-1956-6

Malik M, Camm AJ (1993) Components of heart rate variability-what they really mean and what we really measure. Am J Cardiol 72(11):821-822

Mukai CN, Lathers CM, Charles JB, Bennett BS, Igarashi M, Patel S (1991) Acute hemodynamic responses to weightlessness during parabolic flight. J Clin Pharmacol 31(10):993-1000, DOI 10.1002/j.1552-4604.1991.tb03662. $\mathrm{x}$

Orini M, Bailón R, Laguna P, Mainardi LT, Barbieri R (2012) A multivariate time-frequency method to characterize the influence of respiration over heart pe- 
riod and arterial pressure. EURASIP Journal on Advances in Signal Processing 2012(1):1-17, DOI 10.1186/ 1687-6180-2012-214

Pan J, Tompkins WJ (1985) A real-time QRS detection algorithm. IEEE Trans Biomed Eng 32(3):230-236, DOI 10.1109/TBME.1985.325532

Parati G, Saul JP, Di Rienzo M, Mancia G (1995) Spectral analysis of blood pressure and heart rate variability in evaluating cardiovascular regulation : A critical appraisal. Hypertension 25(6):1276-1286, DOI 10.1161/01.HYP.25.6.1276

Parati G, Ochoa JE, Lombardi C, Bilo G (2013) Assessment and management of blood-pressure variability. Nat Rev Cardiol 10:143-155, DOI 10.1038/nrcardio.2013.1

Pletser V, Winter J, Duclos F, Bret-Dibat T, Friedrich U, Clervoy JF, Gharib T, Gai F, Minster O, Sundblad P (2012) The first joint european partial-g parabolic flight campaign at moon and mars gravity levels for science and exploration. Microgravity Sci Tec 24(6):383-395, DOI 10.1007/s12217-012-9304-y

Pump B, Videbæk R, Gabrielsen A, Norsk P (1999) Arterial pressure in humans during weightlessness induced by parabolic flights. J Appl Physiol (1985) 87(3):928

Seps B, Beckers F, Aubert AE (2002) Heart rate variability during gravity transitions. In: Comput Cardiol, IEEE, vol 29, pp 433-436, DOI 10.1109/CIC.2002.1166802

Sides MB, Vernikos J, Convertino VA, Stepanek J, Tripp LD, Draeger J, Hargens AR, Kourtidou-Papadeli C, Pavy-LeTraon A, Russomano T, et al (2005) The bellagio report: cardiovascular risks of spaceflight: implications for the future of space travel. Aviat Space Environ Med 76(9):877-895

Summers RL, Martin DS, Platts SH, Mercado-Young R, Coleman TG, Kassemi M (2010) Ventricular chamber sphericity during spaceflight and parabolic flight intervals of less than $1 \mathrm{~g}$. Aviat Space Environ Med 81(5):506510, DOI 10.3357/ASEM.2526.2010
Task Force of the European Society of Cardiology and the North American Society of Pacing and Electrophysiology (1996) Heart rate variability: standards of measurement, physiological interpretation and clinical use. Eur Heart J 17(2):354-381

Verheyden B, Beckers F, Aubert AE (2005) Spectral characteristics of heart rate fluctuations during parabolic flight. Eur J Appl Physiol 95(5):557-568, DOI 10.1007/ s00421-005-0016-5

Vigo DE, Ogrinz B, Wan L, Bersenev E, Tuerlinckx F, Van den Bergh O, Aubert AE (2012) Sleep-wake differences in heart rate variability during a 105-day simulated mission to mars. Aviat Space Environ Med 83(2):125-130, DOI 10.3357/ASEM.3120.2012

Vigo DE, Tuerlinckx F, Ogrinz B, Li W, Simonelli G, Bersenev E, Van den Bergh O, Aubert AE (2013) Circadian rhythm of autonomic cardiovascular control during mars500 simulated mission to mars. Aviat Space Environ Med 84(10):1023-1028, DOI 10.3357/ASEM.3612. 2013

Wan L, Ogrinz B, Vigo D, Bersenev E, Tuerlinckx F, Van den Bergh O, Aubert AE (2011) Cardiovascular autonomic adaptation to long-term confinement during a 105-day simulated mars mission. Aviat Space Environ Med 82(7):711-716, DOI 10.3357/ ASEM.2986.2011

Widjaja D, Varon C, Caicedo Dorado A, Suykens JAK, Van Huffel S (2012) Application of kernel principal component analysis for single-lead ecg-derived respiration. IEEE Trans Biomed Eng 59(4):1169-1176, DOI 10.1109/TBME.2012.2186448

Widjaja D, Vandeput S, Van Huffel S, Aubert AE (2013) Gravity effects on heart rate variability during partial gparabolic flights. In: Proc. of the 19th IAA Humans in Space Symposium

Widjaja D, Caicedo Dorado A, Vlemincx E, Van Diest I, Van Huffel S (2014) Separation of respiratory influences from the tachogram: a methodological evaluation. PLoS One 9(7):e101,713, DOI 10.1371/journal.pone.0101713 